\title{
Application of CAE to Model and Simulate Wheel-ground Contact in Railway Vehicle
}

\author{
Julian Malaka \\ Faculty of Mechanical Engineering, Silesian University of Technology, Konarskiego 18A, 44-100 Gliwice, Poland \\ julian.malaka@polsl.pl
}

SNE 31(3), 2021, 127-132, DOI: 10.11128/sne.31.tn.10573 Received: March 10, 2021 (Selected EUROSIM 2019 Postconf. Publ.); Revised: August 24, 2021; Accepted: August 30, 2021 SNE - Simulation Notes Europe, ARGESIM Publisher Vienna, ISSN Print 2305-9974, Online 2306-0271, www.sne-journal.org

Abstract. Drive is one of the most vital elements of technological machines, transport equipment and vehicles. Its selection process is to ensure appropriate motion parameters, that it why mechanical analysis is crucial. Modern CAE (Computer Aided Engineering) tools facilitate and accelerate designers' work, but they can also be misleading if obtained results are misinterpreted. Scepticism with regard to computer simulations is indispensable so as not to hastily recognise something as an ultimate solution to a given problem hastily. In the software based on the visualisations of designed systems, a user is usually not able to influence the motion-describing equations and the method of solving them directly. Most results can yet be evaluated on the basis of the mathematical descriptions of systems under consideration.

This is the procedure which I used to conduct the verification and validation of a model created with the application of a CAE tool. In the article I described the object of the research, the process of its preparation and analysis as well as the results which eventually determine the usefulness of the selected technique in the modelling of vehicle drive mechanics. I named the problems related to the creating of simulations and I suggested solutions to them. The activities carried out provide information helpful in the correct use of the tools that facilitate calculations as well as in the interpretation of the results.

\section{Introduction}

The multitude of phenomena which may accompany the contact of rigid bodies results in the complexity of the contact modelling and simulation.
In order to perform the calculations it is necessary to know the parameters determined experimentally in a given combination of materials and geometry. One should also perform the preliminary assessment of the factors which influence the behaviour of modelled objects, and know the approximate course of the simulation. The computer analysis of the deformation of bodies and the dynamics of their contact requires high computing power, which is why only the phenomena of the actual significance are taken into consideration.

I suggest the use of the dynamic simulation method, which is available in CAD program and which can be easily and swiftly apply in the design process. The objective of the research described in the publication was to verify and analytically validate the model of the wheel and suspension system of a railway vehicle, created in the CAE environment. By the verification I mean the determination whether the implementation of a model is in accordance with the assumptions and allows to show specific features of an object. The validation is to determine whether a model in a given field of application accurately reflects the reality $[1,2,3,4]$. The considerations to the issue in question lead to the development of knowledge in respect of the techniques of the drive system simulation. It is particularly vital in the process of the virtual prototyping of vehicles, transport equipment or technological machines. The fast and adequately accurate numerical analysis of the behaviour of objects enables the streamlining of the design process and the reduction of the adverse event risks in the reality.

In the simulation of the system under consideration it is important to present the events resulting from the wheel-ground contact. It is known that if a wheel is driven at too high acceleration, the slip occurs. The limit value of the acceleration can be easily determined analytically. The phenomenon was observed in an actual, physical model of a vehicle. 


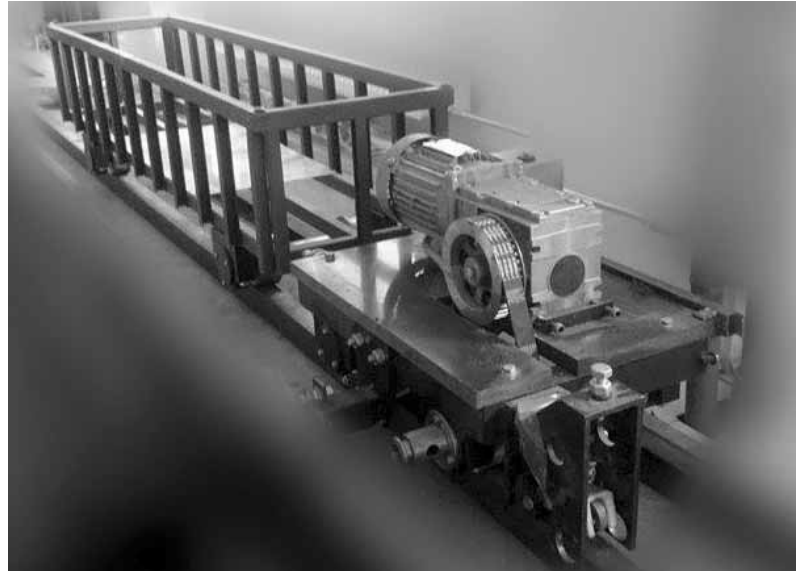

Figure 1: View of physical model of system under consideration.

The research is to demonstrate whether the CAE model enables its representation with a high degree of accuracy. The solution can be assessed on the basis of the mathematical description of the simple case under consideration. The use of the suggested technique in the analysis of complex mechanisms is conditioned by obtaining the satisfactory results of the simulation in question. I adopted the hypothesis that the limit value of the acceleration conditioning the traction of the driving wheels which results from the simulation corresponds to the value calculated analytically. The appropriate preparation of the object and the station in the nature of the model created in the CAE software and the tools for the analysis of the simulation data enables the verification of the assumptions. This way, one can assess the usefulness of given solutions in the modelling of the phenomenon described, as well as identify problems and the key aspects in the process of creating its computer simulation.

\section{Methods}

The system under consideration was represented in the CAE environment in order to enable the verification of the chosen modelling method. I conducted the validation research through the comparison of the results of the computer simulation with those of the analytical calculations. For this purpose it was necessary to formulate mathematical formula and substitute relevant number values into it.

\subsection{Mechanical analysis}

Certain assumptions pertaining to the conditions of the operation of the physical system under consideration were adopted.
No additional slip-increasing elements (such as water, lubrication, contamination) occur during the drive, and the forces acting on individual bodies result from the distribution of the mass of the object and the front axle drive.

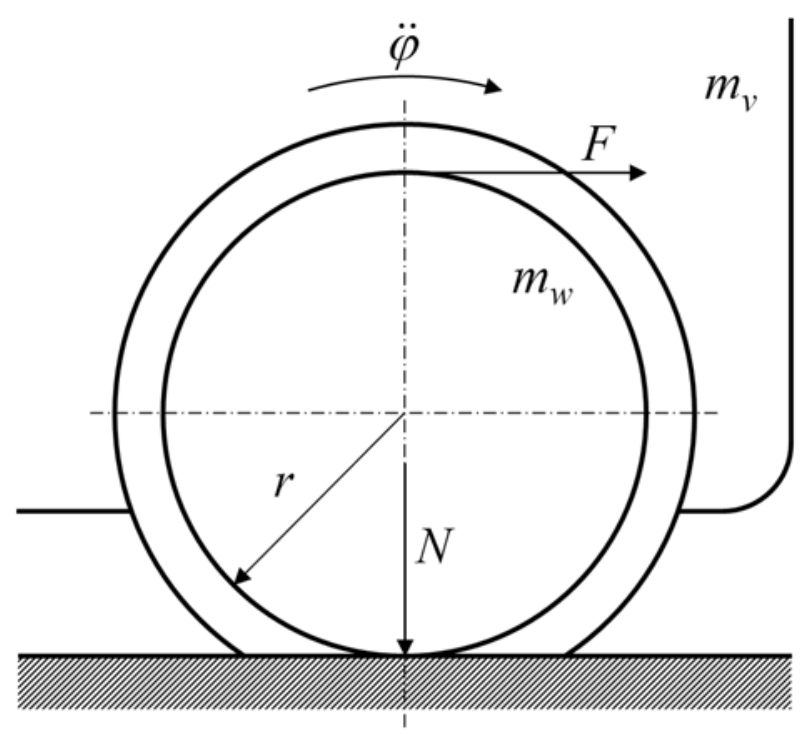

Figure 2: Mechanical diagram of vehicle drive system.

The object under consideration is presented in Figure 2, where:

$\ddot{\phi}$ - angular acceleration of vehicle wheels $\left[\frac{\mathrm{rad}}{\mathrm{s}^{2}}\right]$,

$F$ - resultant force on the circumference of wheels $[N]$,

$m_{w}$ - mass of one set of wheels $[\mathrm{kg}]$;

$m_{v}$ - mass of vehicle, sitting on wheels $[\mathrm{kg}]$.

$N$ - pressure force in the point of wheel-ground contact $[N]$,

$r-$ vehicle wheel radius $[m]$.

The mechanical analysis enables to formulate the mathematical description of the system under consideration and determine the anticipated values of individual parameters. In the case under consideration it is the limit value of the angular acceleration of the wheels $\ddot{\phi}$ with respect of their traction to the ground. It was assumed in the calculations that the $\ddot{\phi}$ value was constant. This way the electric drive accelerates the vehicle in the physical model of the system.

The results of the calculations served as a model of the actual behaviour of the examined object. They constitute the reference during the validation of the model and the simulation being the subject matter of the research. 


\subsection{Modelling and CAE simulation}

The examined model was created in the Siemens NX program. This environment enables the definition of the geometry and the physical features of the individual elements constituting the vehicle and the ground, their kinematic relations, parameters of the contact of the bodies and drive sources.

a)
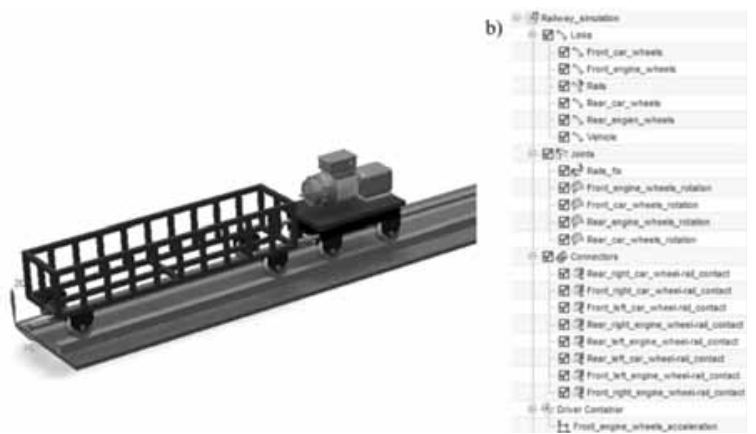

Figure 3: Siemens NX program windows with the definition of the parameters of the model: a) system geometry, b) links, kinematic joints, body contact and drive.

In order to perform the dynamic analysis in the chosen software, Multi-Body Dynamics (MDB) RecurDyn-type CAE tool was used; it is based on the ordinary differential equations and relative coordinates [6].

Conducting the simulation allows to generate the charts of the physical quantity course in the system under consideration as well as to visualise its operation in the form of a 3D animation. This allows to first verify the model, determining whether the data resulting therefrom are as expected and useful in the analysis of the contact of the drive wheels with the ground. The virtual object should behave in a way that is close to the actual one and show its specific features. In the created simulation the instantaneous value of the rotational velocity of a chosen wheel as well as other parameters can be read.

In the drive defining window the constant angular acceleration of a given rotational joint is entered - in the case under consideration it pertains to the rotation of the drive wheels in the bearing support. After the defined value is exceeded, the drive wheel slip should occur, causing the wheels turning with the velocity higher than the one of the wheels actuated by pulling the vehicle. The comparison of the course of the velocity of two elements - the drive wheel and the driven wheel - constitutes the basis for the identification of a slip in the examined system.
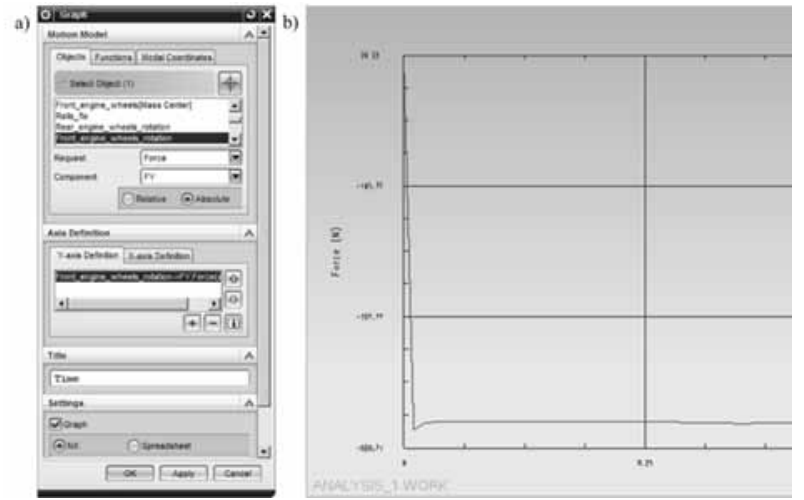

Figure 2: Chart definition window in the simulation in Siemens NX program (a) and an excerpt from the chart presenting the value of the pressure force of driving wheels on the ground (b).

The model accurately presents the reality providing the difference of the velocity occurs after there was defined the angular acceleration exceeding its limit value determined in the analytical calculations. By checking this condition one could evaluate the validity of the assumed hypothesis.

\section{Results}

The obtained results pertained to the mathematical description of the mechanical case under consideration and the simulation of the system in the chosen CAE environment. They made it possible to conduct the verification and validation of the analysed model of the railway vehicle in respect of the representation of the wheel-ground contact.

\subsection{Results of mechanical analysis}

The dynamic equation of the motion of the drive wheels had the following form (1).

$$
J_{\text {red }} \cdot \ddot{\phi}=r \cdot F
$$

In terms of retaining the traction, the cylindrical surface of wheels and the ground surface did not move with respect to each other.

The angular velocity of the driving wheels $\dot{\phi}$ is then is then fully translated into the linear velocity of the vehicle $\dot{x}$, expressed in equation (2).

$$
\dot{x}=r \cdot \dot{\phi}
$$


On the basis of (2) and the relation between the kinetic energy and the mass moment of inertia, I calculated the mass moment of the inertia of the vehicle reduced to the vehicle wheels.

$$
J_{\text {red }}=4 \cdot J_{w}+\left(4 \cdot m_{w}+m_{v}\right) \cdot r^{2},
$$

where $J_{w}$ was a mass moment of inertia of one set of wheels $\left[\mathrm{kg} \cdot \mathrm{m}^{2}\right]$.

The resultant force acting at the circumference of the drive wheels could be calculated by conversion (4).

$$
F=\frac{J_{r e d} \cdot \ddot{\phi}}{r}
$$

The slip of drive wheels did not occur as long as the force $F$ did not exceed the stiction threshold $T$. Its value depended on the pressure $N$ in the point of contact of the bodies and on the stiction coefficient $\mu$ between the wheel and ground materials. This was expressed in equation (5).

$$
T=\mu \cdot N
$$

The traction limit is the situation of balancing the driving force $F$ and the stiction threshold $T$. The conversions (6) resulted in a formula for the maximum angular accelera-

\begin{tabular}{|c|c|c|c|}
\hline Quantity & Symbol & Unit & Value \\
\hline $\begin{array}{l}\text { Mass moment of inertia of } \\
\text { one set of wheels }\end{array}$ & $J_{w}$ & $\mathrm{~kg} \cdot m^{2}$ & 0.0035 \\
\hline Mass of one set of wheels & $m_{w}$ & $\mathrm{~kg}$ & 4.4100 \\
\hline $\begin{array}{l}\text { Mass of vehicle, sitting on } \\
\text { wheels }\end{array}$ & $m_{v}$ & $\mathrm{~kg}$ & 141.7200 \\
\hline $\begin{array}{l}\text { Pressure in the point of the } \\
\text { contact of drive wheels with } \\
\text { the ground }\end{array}$ & $N$ & $N$ & 511.6000 \\
\hline Vehicle wheel radius & $r$ & $m$ & 0.0420 \\
\hline $\begin{array}{l}\text { Coefficient of wheels- } \\
\text { ground stiction } \\
\text { (steel on steel) }\end{array}$ & $\mu$ & - & 0.3000 \\
\hline $\begin{array}{l}\text { Permissible angular } \\
\text { acceleration of drive wheels }\end{array}$ & $\ddot{\phi}_{\max }$ & $\frac{\mathrm{rad}}{s^{2}}$ & $\underline{21.8100}$ \\
\hline
\end{tabular}
tion of the drive wheels taking into account the adopted conditions.

Table 1: Data and results of mechanical analysis.

$$
\begin{gathered}
F_{\text {max }}=T \Rightarrow \\
\Rightarrow \frac{J_{\text {red }} \cdot \ddot{\phi}_{\text {max }}}{r}=\mu \cdot N \Rightarrow \\
\Rightarrow \ddot{\phi}_{\text {max }}=\frac{\mu \cdot N \cdot r}{J_{\text {red }}}
\end{gathered}
$$

Using the formulas presented, I determined the permissible angular acceleration of the drive wheels, the exceeding of which resulted in the occurrence of slip.

Data and results are collated in Table 1. The obtained value $\ddot{\phi}_{\text {max }}$ constituted the reference in the model validation process.

\subsection{Simulation results}

After the model had been adjusted, I conducted the simulation of the drive and generated the charts of the velocity of the drive wheel and the driven wheel at a defined acceleration.

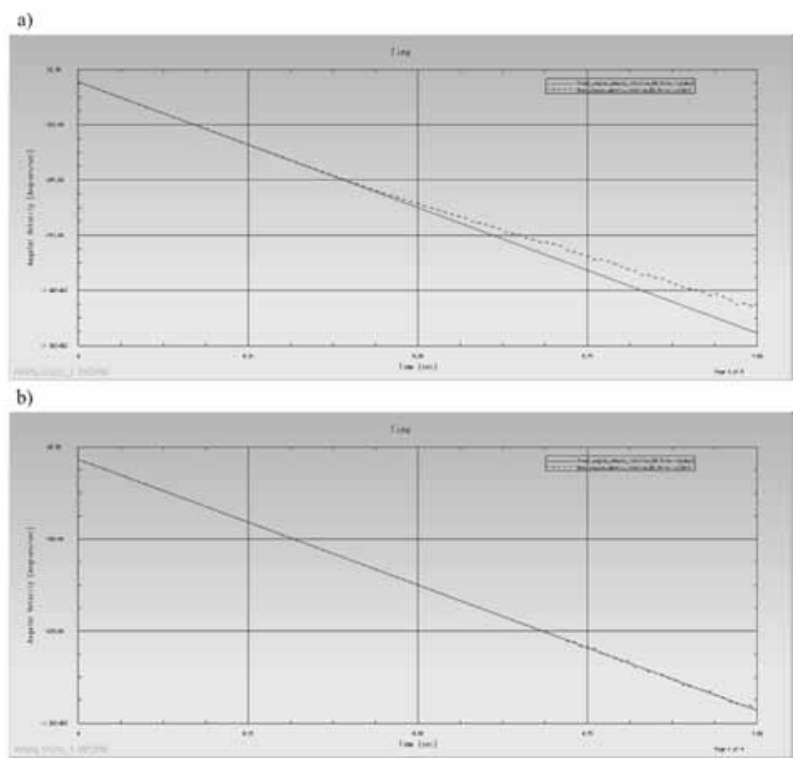

Figure 5: Charts of velocity of analysed wheels:

a) at the defined acceleration equalling $23 \frac{\mathrm{rad}}{\mathrm{s}^{2}}-$ visible velocity divergence,

b) at the defined acceleration equalling $22 \frac{\mathrm{rad}}{\mathrm{s}^{2}}-$ convergent velocities.

I observed what happened to the system when the value of the acceleration defined in the simulation was lower than the calculated one, when it equalled to it and exceeded it. I conducted a set of trials at various acceleration values. It allowed to indicate the highest of the examined values of this parameter, at which the divergence between the analysed velocities did not occur yet. 


\begin{tabular}{lll}
$\begin{array}{l}\text { No. } \\
\text { of } \\
\text { trial }\end{array}$ & Defined acceleration $\left[\frac{\mathrm{rad}}{\mathrm{s}^{2}}\right]$ & Discrepancy of velocity \\
\hline $\mathbf{1}$ & 22.00 & No \\
\hline $\mathbf{2}$ & 23.00 & Yes \\
\hline $\mathbf{3}$ & 22.34 & No \\
\hline $\mathbf{4}$ & 22.50 & No \\
\hline $\mathbf{5}$ & 22.70 & Yes \\
\hline $\mathbf{6}$ & 22.60 & Yes \\
\hline $\mathbf{7}$ & 22.55 & No
\end{tabular}

Table 2: Simulation results.

Table 2 presents the results of the simulations during the trials at various values of the defined angular acceleration of the vehicle drive wheels.

\section{Discussion and Conclusions}

What stems from the conducted simulation trials is that the divergence of the rotational velocity of the drive wheel and the driven wheel in the modelled vehicle occurs when the threshold of the angular acceleration, equal to $22.55 \frac{\mathrm{rad}}{\mathrm{s}^{2}}$, is exceeded.

According to the adopted assumptions and the method of the analysis of the simulation data, this is the limit value at which the slip does not occur. The value resulting from the analytical calculations was equal to $21.81 \frac{\mathrm{rad}}{\mathrm{s}^{2}}$. It means that the inconsistency between the numerical model, created in the chosen CAE environment, and the mathematical model is approx. $3 \%$.

On the basis of such a low level of error, it can be ascertained that the validation of the suggested solution gives a positive result. Minor discrepancies may result from rounding the values in calculations and averaging the values read from the charts throughout the simulation. The results of the research demonstrate that the modelled object behaves in a way consistent with the mathematical description, which confirms the adopted hypothesis.

\subsection{Problems during verification of the model}

In order to present the dynamic features of the examined system, in the model there was defined the spatial contact of rigid bodies between each of the vehicle wheels and the rails on which the wheels roll.
The software documentation [5] contains the guidelines concerning the parameters of the contact taking into consideration the materials of which the objects are made. After the application thereof, it turned out that the model did not show what had been expected. In the simulation the vehicle did not follow the expected motion path after the angular acceleration of the drive wheels had been defined. Strong bounces of the contacting surfaces occurred and the object was "thrown" out of the stage.

The major problem was the geometric form of the objects coming into contact with each other. When the convex object is located inside the concave object, their angles should not be closely adjacent to each other. This can be remedied by rounding off sharp convex edges in the geometric model [5]. In the case under consideration this situation occurred in the point of the contact of a wheel edge and the rail. The correction of the model, as shown in Figure 6, was made. a)

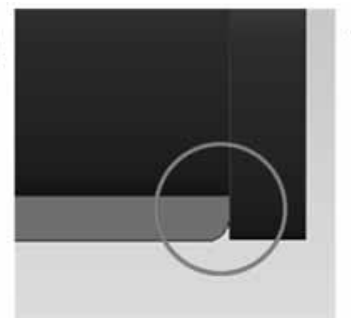

b)

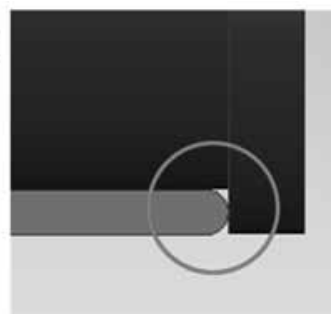

Figure 6: Point of contact of rail and wheel angle in vehicle model: a) before geometry correction, b) after geometry correction.

On the basis of the observation of the visualisation of the vehicle motion it was stated that once the correction had been made the modelled object behaved as expected.

In order to perform analytical calculations, the value of the pressure force in the point of the contact of the drive wheels and the ground was necessary. Once the simulation begins, the force should stabilise at a constant level when the vehicle is at rest (only gravity acts on it). When the default accuracy of the simulation and the contact parameters proposed in the documentation were used, the generated course of the pressure force value was strongly turbulent. It resulted from the unevenness of the objects, causing their motion with respect to each other, which was visible in the simulation at a large zoom.

The unevenness occurred even though the contact surfaces had been defined as smooth, so they should be perfectly adjacent to each other. The model was finetuned by adjusting the individual parameters according to the guidelines in the software documentation. 
Table 3 presents the parameters which were changed from the default values.

\begin{tabular}{lll}
\hline Parameter & $\begin{array}{l}\text { Default value / Value sug- } \\
\text { gested in software docu- } \\
\text { mentation }\end{array}$ & $\begin{array}{l}\text { Correct value } \\
\text { determined } \\
\text { experimentally }\end{array}$ \\
\hline Stiffness & 100000.0 & 10000.0 \\
\hline $\begin{array}{l}\text { Maximum } \\
\text { Facet Size }\end{array}$ & 2.0 & 0.5 \\
Factor & & 1.0 \\
\hline $\begin{array}{l}\text { Plane Toler- } \\
\text { ance Factor }\end{array}$ & 3.0 & \\
\hline
\end{tabular}

Table 1: Wheel-ground contact parameters of model fine-tuning.

Once the experimentally defined parameters had been applied, the model met expectations and the simulation returned the values needed for the research.

\subsection{CAE modelling in view of other methods of mechanical analysis}

The CAE tools are an integral part of numerous designassisting computer programs. They enable to create with ease a dynamic simulation on the basis of the geometrical model of a designed system.

In this way, neither is it necessary to build or transform equations of motion not to write a code to solve them. The methods of numerical calculations are implemented in the CAE environment. Its operation mostly boils down to defining the physical parameters of the elements of the analysed mechanism. In the chosen software ordinary differential equation and relative coordinates are applied instead of differential algebraic equations with global coordinates. This reduces the number of equations and the time needed to solve them [6].

The validation and verification of the model allowed me to select appropriate simulation settings based on the mathematical analysis. The model correctly reflecting a given phenomenon in one case with a high probability will also work in others - more complicated but similar in respect of conditions. In addition to the numerical results, the visualization of the operation of the examined system is also helpful in the analysis of solutions created.
The techniques of the preparation as well as the verification and validation of the numerical model suggested in the article improve the device prototyping process and make it more accurate. It is particularly essential for the development of modern means of transport. Modern environmental and market requirements lead to their optimisation with regard to energy consumption and motion parameters $[7,8]$. The simulation calculations in the CAE environment may facilitate the choice and configuration of drives as well as predicting their operation, helping to avoid unwanted situations in reality.

\section{References}

[1] Balci O. Verification, validation, and certification of modeling and simulation applications. In: Proceedings of the 2003 Winter Simulation Conference, pp. 150-158. New Orleans, USA, December 2003.

[2] Karkula M. Weryfikacja i walidacja dynamicznych modeli symulacyjnych procesów logistycznych. Logistyka 2, pp. 717-726 (2012).

[3] Sargent R G. Verification and validation of simulation models. In: Proceedings of the 2011 Winter Simulation Conference, pp. 183-198. Phoenix, USA, December 2011.

[4] Schlesinger S, Crosbie R E, Gagné R E, Innis G S, Lalwani C S, Loch J, Sylvester R J, Wright R D, Kheir N, Bartos D. Terminology for model credibility. Simulation 3(32), pp. 103-104 (1979).

[5] Siemens Documentation: NX 12 Help. https://docs.plm.automation.siemens.com/tdoc/nx/12/ nx_help, last accessed 2019/06/09.

[6] Using RecurDyn. http://www2.me.rochester.edu/courses/ME204/nx_help/ en_US/graphics/fileLibrary/nx/tdoc_motion/ using_recurdyn.pdf, 2019/06/09.

[7] Walker P D, Abdul Rahman S, Zhu B, Zhang N. Modelling, Simulations, and Optimisation of Electric Vehicles for Analysis of Transmission Ratio Selection. Advances in Mechanical Engineering 2015 (340435), pp. 1-13 (2015).

[8] Yifan D, Yugong L, Wenbo C, Keqiang L. Optimum tyre force distribution for four-wheel-independent drive electric vehicle with active front steering. Int. J. of Vehicle Design 65(4), pp. 336-359 (2014). 